\title{
Caracterización de sistemas de alimentación de ovinos en el departamento de Córdoba, Colombia
}

\author{
Mestra, L.I. ${ }^{1}$.; Santana, M.O. ${ }^{1}$.; Rios, d.A. L. ${ }^{2} ;$ Mejia, L.J. ${ }^{\text {; }}$ Ortiz, C.R. ${ }^{2}$; y Paternina, S.E. ${ }^{1}$.
}

'Corporación colombiana de Investigación Agropecuaria Agrosavia, Centro de Investigación Turipaná. Cereté. Colombia.

${ }^{2}$ Corporación colombiana de Investigación Agropecuaria Agrosavia, Centro de Investigación Tibatitatá. Mosquera. Colombia.

\section{PaLABRAS CLAVE}

Dietas.

Gramíneas forrajeras.

Indicadores Productivos.

Rebaño.

Recursos alimenticios

\section{RESUMEN}

El objetivo de este estudio fue caracterizar los sistemas de alimentación de ovinos y los recursos alimenticios en 26 unidades productivas (UP) de 13 municipios de Córdoba, Colombia. La información se recopiló mediante cuestionarios semiestructurados, conformados por las áreas temáticas: a) Ubicación geográfica de las UP, b) Características de infraestructura y componente animal, c) Manejo animal y control productivo, d) Composición nutricional de alimentos, e) Caracterización de dietas utilizadas y f Manejo de la alimentación. En los análisis descriptivos, se encontró que la mayoría de las UP eran extensivas con predominio de rebaños bovinos y ovinos $(84,6 \%)$, uso de varias razas de ovinos $(61,1 \%)$, verificación del consumo de calostro (88\%), uso de Botrhriocloa pertusa en pastoreo continuo $(69.2 \%)$ y ocasionalmente suplementan los animales $(53.9 \%)$. Con el análisis de conglomerados y la evaluación interna realizada, se encontró que la mejor estructura de agrupamiento se obtuvo con el método de las k medias, el cual concentró las 26 UP en dos grupos G1 y G2, el G1 con 3 UP, caracterizado por rebaños con más de 400 animales, mientras que, el G2 con 23 UP, mostró un inventario promedio menor a 100 animales; ambos grupos con pastoreo continuo y uso de suplementos, con adecuada estructura de los rebaños. Se concluye que, en Córdoba, la producción ovina se basa en el pastoreo, en transición desde un nivel tecnológico medio a semi-intensivo; similar a la producción nacional colombiana, asociada con una inadecuada gestión de los alimentos y con oportunidades de mejora debido a la disponibilidad de recursos alimentarios de buena calidad.

Characterization of sheep feeding systems in the department of Córdoba, Colombia

\section{SUMMARY}

Diets.

Feeding resources.

Grasses.

Herd.

Productive indicators.

\section{INFORMATION}

Cronología del artículo.

Recibido/Received: 11.03.2020

Aceptado/Accepted: 03.08.2020

On-line: 15.10 .2020

Correspondencia a los autores/Contact e-mail:

Imestra@agrosavia.co

\section{INTRODUCCIÓN}

La producción de ovinos en Colombia se caracteriza por el bajo uso de insumos y escasa transferencia de tecnología en todas las fases productivas de la especie (Pastrana-Bonilla, 2002; Arévalo-Garay y CorreaAssmus, 2013). Generalmente, su estructura está asociada a sistemas tradicionales de producción, donde las familias campesinas poseen ovinos como parte de su actividad pecuaria, favoreciendo principalmente el consumo interno de carne en la unidad productiva, así como el comercio local, por lo que son poco im- portantes en su aporte al producto interno bruto, pero tienen gran impacto en la economía y alimentación campesina. Al respecto, Owen et al., (2004) indican que el desarrollo de la ganadería mundial de la especie ovina es clave para la seguridad alimentaria de muchos pequeños productores.

En el ámbito nacional, la región Caribe Colombiana registra la existencia de 1.449.705 ovinos los cuales corresponden al $68,3 \%$ del inventario del país, todos con manejo de tipo extensivo. Las mayores poblaciones de la especie se concentran en los departamentos de la Guajira (42,1\%), Magdalena (11,8\%), Cesar (8,8\%), en el 
Caribe seco con aproximadamente $63 \%$ de la población total de ovinos, y Córdoba $(5,4 \%)$ en el Caribe húmedo, con una población de 90,912 ovinos (ICA, 2020), ubicados en su mayoría en sistemas de producción de pequeños y medianos productores, con bajo nivel tecnológico los cuales juegan un papel preponderante en la economía familiar (Acero-Plazas, 2014)los sistemas pecuarios o sistemas productivos están enfocados hacia las especies con mayor "potencial" de exportación o de mayor consumo nacional, las cuales son: aves (carne, huevos, genética. Así mismo, ante la creciente demanda de proteína animal por la población humana, existe la necesidad de mejorar la productividad de las ovejas a través de la cría, conservación y utilización sostenible de esta especie, para mejorar niveles de vida de los ganaderos y aliviar la pobreza entre los habitantes rurales.

A nivel más específico lo que se puede observar en los sistemas de producción de ovinos en la región caribe es el desconocimiento total o parcial de la composición, disponibilidad y manejo adecuado de los recursos alimenticios de origen forrajero, lo que lleva a una alta utilización de insumos externos como alimentos concentrados comerciales, aumentando los costos de producción (Pugh y Baird, 2012). Esto se evidencia en las demandas de la cadena ovino-caprina del departamento de Córdoba establecidos mediante el Programa Estratégico de Ciencia tecnología e Innovación Agropecuaria (PECTIA 2017-2027).

Bajo este escenario, la implementación de un programa integral de producción de ovinos requiere de información relacionada con todos los componentes del sistema de producción (características del suelo, categorías de alimentos disponibles, valor nutricional y requerimientos de la especie), la cual es insuficiente en la actualidad (Montes, Pérez y de la Ossa, 2016) y nivel de desarrollo tecnológico. Al respecto de desarrollo tecnológico precario, Grajales, Moreno y Atuesta-Bustos (2011) argumentan que en la mayoría de regiones del mundo estos sistemas de producción se encuentran en países pobres por lo que no se dispone de muchos recursos económicos para realizar estudios de investigación que conduzcan al desarrollo de sistemas más productivos. En general de acuerdo con el grado tecnológico los sistemas ovinos se clasifican de la siguiente manera, 1) sistemas extensivos, 2) sistemas semi intensivos, 3) sistemas intensivos y 4) sistemas integrados con cultivos agrícolas.

El estudio general de suelos y zonificación de tierras del departamento de Córdoba (IGAC, 2009) señala que solo $10,5 \%$ de los suelos del departamento tienen vocación de uso para la ganadería (260.662 ha), mientras que $39,7 \%$ tiene vocación agroforestal y 23,6\% agrícola. Estas áreas con vocación ganadera presentan suelos con limitaciones moderadas para el desarrollo de agricultura semi-intensiva e intensiva, pero pueden tener en cambio, uso para el pastoreo extensivo $(47,5 \%)$ y semi-intensivo (52,5\%).

En Colombia, la cadena ovina ha tenido avances aislados, el gobierno nacional ha financiado proyectos para el fomento y adquisición de pie de cría de ovinos y se han desarrollado investigaciones regionales sobre indicadores productivos de importancia económica, en áreas como, genética y alimentación (Vergara-Garay et al., 2016 y Vergara-Garay et al., 2017) y evaluaciones con algunos suplementos alimenticios (Maza-Angulo et al., 2015). En el aspecto de alimentación se requieren más estudios en ovinos, debido a la gran diversidad en la oferta de recursos disponibles y la calidad de los mismos a nivel regional, ya que el valor nutricional de éstos es variable (Rodríguez-Ramírez et al., 2013). Por otra parte, otros autores (González-Garduño, TorresHernández y Arece-García, 2010), afirman que existe escasa vinculación con la agroindustria, la cual podría fortalecerse mediante la integración de esta en programas de alimentación animal sostenibles y adaptados a las condiciones específicas de cada región, contribuyendo así a generar incrementos en la productividad de la especie ovina. Por lo anterior, el objetivo del presente estudio fue caracterizar los sistemas de alimentación de ovinos y la calidad nutricional de los recursos alimenticios disponibles en 26 unidades productivas (UP), del departamento de Córdoba, con el propósito de generar una línea base de información que contribuya a mejorar el conocimiento en esta área, para fortalecer el eslabón primario de la cadena productiva de ovinos en la región caribe colombiana.

\section{MATERIAL Y MÉTODOS}

\section{DESCRIPCIÓN DEL ÁREA DE ESTUDIO Y DE UNIDADES PRODUCTIVAS (UP):}

El departamento de Córdoba está situado en el noroeste de Colombia, a orillas del Mar caribe, con una extensión de 23.980 kilómetros cuadrados representando el $2,1 \%$ del territorio nacional y se encuentra localizado entre los $09^{\circ} 26^{\prime} 16^{\prime \prime}$ y $07^{\circ} 22^{\prime} 05^{\prime \prime}$ de latitud norte, y los $74^{\circ} 47^{\prime} 43^{\prime \prime}$ y $76^{\circ} 30^{\prime} 01^{\prime \prime}$ de longitud oeste. La temperatura media mínima en el departamento es de $22,7^{\circ} \mathrm{C}$ y media máxima de $32,2^{\circ} \mathrm{C}$ (Claro, 1993 citado por Palencia-Severiche, Mercado-Fernández y Combatt-Caballero, 2006).

El promedio anual de la humedad relativa es $84 \%$ y se asocia con procesos de evapotranspiración que varían de suroccidente a nororiente (IGAC, 2009). Respecto a las características físicas del departamento, está dividido en treinta municipios los cuales presentan una regionalización ambiental que se encuentra definida en seis subregiones, que corresponden al Alto Sinú, Sinú medio, Bajo Sinú, Sabana, San Jorge y Zona Costanera. Según la clasificación de las de las zonas de vida predominantes en el Caribe colombiano (Holdridge, 1967), estas subregiones pertenecen a tres zonas climáticas: Bosque seco tropical (Sinú medio, Bajo Sinú, Sabana y Zona Costanera), transición entre Bosque seco y húmedo tropical (San Jorge) y Bosque húmedo tropical (Alto Sinú).

\section{TAMAÑO DE LA MUESTRA Y REALIZACIÓN DE ENCUESTAS:}

Para determinar el tamaño de la muestra (n), se consideró como objetivo la estimación de la proporción de UP correspondientes a pequeños productores en el departamento de Córdoba, en este sentido, el marco muestral fue construido con 85 UP distribuidas en las subregiones del departamento de Córdoba y que estu- 
vieran registradas en las asociaciones local y nacional, Asovicor y Asoovinos, respectivamente, mientras que la determinación del número (n) de UP a encuestar se determinó por la siguiente formula (Aguilar-Barojas, 2005):

$$
\mathrm{n}=\left(\mathrm{N}^{*} \mathrm{Z}^{2 *} \mathrm{p}^{*} \mathrm{q}\right) /\left(\mathrm{d}^{2 *}(\mathrm{~N}+1)+\mathrm{Z}^{2 *} \mathrm{p}^{*} \mathrm{q}\right)
$$

Donde:

\section{$\mathrm{n}=$ tamaño de la muestra}

$\mathrm{N}=$ tamaño de la población

$\mathrm{Z}=$ valor critico de distribución normal estándar a un nivel de confianza dado

p=proporción en la población que cumple la característica objetivo

$\mathrm{q}=1-\mathrm{p}$, proporción en la población que no cumple la característica objetivo

\section{d=error de estimación aceptado}

La captura de la información y colecta de las muestras de alimentos que consumen los ovinos, se realizó durante los meses de marzo de 2017 a junio de 2018, incluyendo de esta manera, todas las épocas del año, lo cual permitió obtener mayor variedad de estos para su valoración nutricional.

Para la aplicación de las encuestas semi-estructuradas, se utilizó la metodología del World mapping of animal feeding systems in the dairy sector (FAO e IDF, 2014), la encuesta estuvo conformada por las siguientes áreas temáticas: a) Ubicación geográfica de las UP, b) Características de la infraestructura y el componente animal, c) Manejo animal y control productivo, d) Composición nutricional de los alimentos, e) Caracterización de las dietas utilizadas y f) Manejo de la alimentación. La información se estructuró y se clasificaron las variables de mayor interés para tipificar y caracterizar los sistemas de alimentación y los recursos alimenticios que se utilizan.

\section{CARACTERIZACIÓN DE LAS ESPECIES FORRAJERAS Y CALIDAD NUTRICIONAL}

La colecta de los recursos forrajeros y subproductos agroindustriales (cereales y aceiteros) utilizados en la alimentación de los ovinos, se realizó al momento de aplicar las encuestas de caracterización de las UP, recolectándose 140 muestras de los forrajes que los ovinos consumían (verificadas por observación en campo), las cuales se clasificaron en las siguientes categorías: gramíneas forrajeras $(\mathrm{GF})=58,57 \%$; leguminosas forrajeras $(\mathrm{LF})=3,57 \%$; árboles forrajeros y arbustos no leguminosos $(\mathrm{AFy} A N L)=10,0 \%$; otros forrajes $(\mathrm{OF})=$ $5,71 \%$; granos $(\mathrm{GR})=3,57 \%$; plantas oleaginosas y subproductos $(\mathrm{POySPR})=4,3 \%$; raíces, tubérculos y subproductos $($ RTySPR $)=0,71 \%$; frutas de leguminosas y subproductos (FLySPR) $=5,0 \%$ y mezcla de ingredientes $(\mathrm{MI})=8,57 \%$, de esta manera quedaron disponibles los resultados para consulta de asistentes técnicos y productores en el momento que los requieran para la formulación de planes de alimentación para el rebaño.
Las muestras de GF se obtuvieron de los potreros dedicados al pastoreo de los ovinos, antes del ingreso de los animales a estos, mediante el método de simulación de pastoreo "Hand plucking", se tomó una muestra de un kilogramo, pesada en balanza electrónica digital Ohaus ® V11P6. La obtención de muestras de LF, AFyANL se realizó mediante la metodología propuesta por Mahecha et al., (2000), la cual consistió en seleccionar tres estratos o arbustos en el potrero para recolectar todas las hojas y tallos tiernos presentes en cada uno de los arbustos. Las muestras de POySPR y RTSPR disponibles en las UP, se obtuvieron utilizando muestreadores para solidos (lanza metálica) en sacos cerrados que fueron muestreados en su totalidad, cuando el lote de sacos fue inferior a 10 unidades, y en lotes con mayores cantidades se muestreo usando la metodología de "quartering" descrita por Herrman (2001). Posteriormente, las muestras fueron sometidas a secado en estufa de ventilación forzada en el laboratorio del Centro de Investigación Turipaná, durante $48 \mathrm{~h}$ a $60^{\circ} \mathrm{C}$. Luego estas fueron molidas en un molino Wiley Mill, utilizando una criba de $1.0 \mathrm{~mm}$.

Las categorías de los recursos alimenticios disponibles en las unidades productivas se establecieron usando como referencia la plataforma AlimenTro (Agrosavia, 2020), la cual es una herramienta que está a disposición de productores y público en general, para la consulta y diseño de dietas para los animales. El análisis de la composición nutricional de los forrajes y demás recursos alimenticios se realizó usando un espectrómetro FOSS NIRSystems modelo DS2500. Los espectros se registraron con WinISI 4.7.0 (FOSS). Cada muestra se midió tres veces y el promedio de los espectros replicados, obtenidos como log (1 / R) se utilizaron para interpolarlos en las ecuaciones de predicción desarrolladas previamente para los macrocomponentes: materia seca (MS), proteína cruda $\mathrm{PC}$, extracto etéreo (EE), fibra en detergente neutro (FDN), fibra en detergente ácido (FDA), lignina (LIG) (Ariza-Nieto et al., 2018)legume forages, and other forage plants. La evaluación de la energía bruta de los recursos alimenticios se realizó mediante el método de combustión adiabática (Parr Instrument) Método oficial (AOAC International, 1990) y los resultados de análisis se registraron en la plataforma AlimenTro.

\section{ANÁLISIS ESTADÍSTICO}

Para la aplicación de la encuesta semi estructurada, con un nivel de confianza del $97.5 \%$ y un error de estimación en la proporción del 16\%, fueron seleccionadas en forma aleatoria 26 UP de las 85 incluidas en el marco muestral. La información se digitalizó y analizó en el programa estadístico SPSS versión 15.0. mediante estadística descriptiva, a fin de obtener generalidades de las UP evaluadas. Por otra parte, se realizó un análisis de conglomerados empleando la información de las 25 variables, tanto cualitativas como categóricas. El agrupamiento de las 26 UP se realizó utilizando la distancia Euclidiana y los métodos de agrupamiento jerárquico aglomerativo (usando el método de Ward) y el método de las K medias.

Dada la presencia de múltiples variables categóricas en las encuestas aplicadas, se realizó un análisis de 
correspondencia múltiple (ACM) para obtener nuevas variables sintéticas, conformadas por la información de las variables cualitativas iniciales. En este análisis se eligió un número de estas variables sintéticas, de modo tal, que explicaran un porcentaje mínimo de la variabilidad total ( $70 \%$ en este caso). Una vez realizado el ACM, las variables sintéticas retenidas fueron utilizadas para los análisis de clasificación al combinarlas con las variables cuantitativas, sobre esta nueva base de datos, fueron implementados los métodos $\mathrm{K}$ medias y de agrupamiento jerárquico aglomerativo con el objetivo de definir los grupos para las UP encuestadas (Hastie et al., 2009).

La determinación del número óptimo de grupos se realizó por medio de los criterios estadísticos (Hastie et al., 2009), del paquete NbClust de $\mathrm{R}$ (Charrad et al., 2014), basados en la regla de la mayoría, desde la frecuencia obtenida por un número dado de grupos en 30 criterios diferentes considerados en la librería. Para realizar la validación interna de los grupos obtenidos, se empleó el método de la amplitud de silueta promedio de cada método, el número de UP con amplitud de silueta negativa, el índice de Dunn y la correlación cofenética (Valores mayores a 0.75 indican la capacidad del dendograma para representar las distancias originales) para el caso del agrupamiento jerárquico. La edición y análisis de datos se llevaron a cabo utilizando los paquetes $\mathrm{NbClust} \mathrm{(Charrad} \mathrm{et} \mathrm{al.,} \mathrm{2014),} \mathrm{fpc}$ (Hennig, 2015) y factoextra (Kassambara and Mundt, 2020), del programa R (R Core Team, 2017).

\section{RESULTADOS}

De acuerdo con el análisis descriptivo realizado para la caracterización general de las UP, se presentan los siguientes resultados.

\section{Características de las Unidades PROductivas (UP):}

En la Tabla I se pueden observar la ubicación y número de predios caracterizados por subregión y municipio. De las UP estudiadas, el 100\% realizan el ciclo completo productivo (cría, levante y ceba de corderos), para posteriormente comercializar los animales en pie. De estas UP el 73,07 \% tiene un manejo extensivo, representado por el pastoreo de ovinos sobre grandes áreas en tierras marginales, e inapropiadas para otras formas de agricultura; solo el 3,85 \% de las UP tenía manejo intensivo, con inversión en instalaciones y alimentos concentrados para el rebaño, y el 23,07 \% con manejo semi-intensivo que representa la combinación entre el sistema extensivo e intensivo.

Con relación al tamaño de las UP se encontró que el 15,38\% es $<10$ ha, el 30,77\% osciló entre $10-50$ ha, el $23,08 \%$ entre $50-100$ ha y el 30,77\% correspondió a UP con más de 100 ha. Mientras que el área (ha) destinada a la producción de ovinos dentro de las UP evaluadas resultó de la siguiente manera: $<10$ ha el 30,77\%; de 10-50 ha el 42,30\%; de 50-100 ha el 19,23\% y más de 100 ha el $7,7 \%$.

De acuerdo con la altitud sobre el nivel del mar (msnm), las UP se distribuyen de la siguiente forma, el $46,16 \%$ entre $0-50 \mathrm{msnm}$, el $42,31 \%$ entre $50-100 \mathrm{msnm}$ y el $11,54 \%$ a más de $100 \mathrm{msnm}$, indicando que las muestras incluyeron todos los tamaños de las UP y las condiciones de altitud representativas de la zona.

\section{CARACTERÍSTICAS DE LA INFRAESTRUCTURA Y DEL COMPONENTE ANIMAL}

La infraestructura básica que se maneja en $82,3 \%$ de las UP era el aprisco, mientras que el $46,15 \%$ posee bodega para alimentos y solo el $3,85 \%$ estaba provisto de acueducto.

En cuanto al componente animal, dentro de las UP la base genética corresponde a ovinos de pelo colombiano (OPC), conocidos también como "camuros". El OPC se caracteriza por ser una raza que se ha adaptado a las diferentes condiciones geográficas y climáticas de Colombia, demostrando rusticidad, mansedumbre, prolificidad y un excelente comportamiento animal (Arcos et al., 2002). El 61,1\% de las UP posee dos razas, el 28,6\% maneja tres o más razas o cruzamientos y el $10,3 \%$ de los productores maneja una sola raza. Estas razas utilizadas en las UP correspondían a Kathadin $(50 \%)$, Santa Inés $(38,5 \%)$, Dorper $(15,4 \%)$, Pelibuey $(11,5 \%)$ y otros cruces $(11,5 \%)$. Relativo al manejo del rebaño, el 57,7\% de las UP organiza su rebaño en uno o dos lotes de ovinos, lo que impide que los apareamientos sean practicados en forma técnica, adicionalmente, no se registra el peso al nacimiento y en general la identificación y el manejo de los registros son deficientes. Cabe destacar que el $84,6 \%$ de las UP asocian los bovinos con ovinos, mientras que el 15,4\% se dedica a la ovinocultura únicamente. A nivel general, la población ovina de las UP varió entre 26 y 518 ovinos totales, con un promedio de $129 \pm 133$ animales, distribuidos de la siguiente forma: $44 \%$ hembras adultas, 30\% ovinos de levante, $24 \%$ crías y $2 \%$ reproductores.

\section{MANEJO ANIMAL Y CONTROL PRODUCTIVO}

Identificación: En lo referente al manejo animal, el $53,85 \%$ de los productores identifica los ovinos con distintos métodos, como la chapeta, el tatuaje y collares, mientras que el $46,15 \%$ no realiza ninguna identificación de sus animales (Figura 1).

Uso y manejo de registros: $\mathrm{El} \mathrm{77,0 \%} \mathrm{de} \mathrm{los} \mathrm{produc-}$ tores lleva registros; sin embargo, el 23,0\% no los utiliza para la toma de decisiones. El medio más utilizado es el impreso o manuscrito (65\%), digital (20\%), software $(10 \%)$ y varias formas de registros $(5 \%)$. Con relación a la información contenida en los registros, generalmente los productores llevan uno o varios datos productivos, siendo los más comunes el registro de pesaje de los ovinos, inventario y mortalidad. Los pesajes que registran son: al nacimiento, al destete y/o a la venta (Figura 1).

Prácticas de manejo animal: las prácticas más generalizadas son la verificación de toma de calostro en las primeras horas del nacimiento de las crías que la realiza el 88,5\% de los productores; la desinfección de ombligo $(84,7 \%)$, arreglo de pezuñas $(69,2 \%)$, revisión de condición corporal $(42,3 \%)$, esquila $(7,7 \%)$, descole $(11,5 \%)$ y castración $(34,6 \%)$.

Asistencia Técnica: En lo relacionado con la asistencia técnica, el 80,8\% de los productores encuestados consulta a los asistentes técnicos para el manejo sanitario o reproductivo del rebaño. 


\section{DIVERSIDAD Y COMPOSICIÓN NUTRICIONAL DE LAS ESPECIES FORRAJERAS EN LAS UP DEL DEPARTAMENTO DE CÓRDOBA}

Las especies forrajeras y suplementos colectados y analizados en las UP para la alimentación de los ovinos fueron $58,57 \%$ gramíneas forrajeras (GF); $10,0 \%$ árboles forrajeros o arbustos no leguminosos (AF y ANL); 12,15\% de mezcla de ingredientes (MI); 10,71\% otros forrajes (OF), 4,29\% plantas oleaginosas (PO), 3,57\% leguminosas forrajeras (LF), y 0,71\% raíces, tubérculos y subproductos (RT y SPR) . La mayor diversidad de alimentos se logró colectar en las
UP semi-intensivas de los municipios pertenecientes a las subregiones de Medio Sinú, San Jorge y Ciénaga

\section{En la Tabla II.}

Se presenta la composición nutricional de los forrajes utilizados en las UP para la alimentación de los ovinos, evidenciándose que las gramíneas forrajeras (GF) y los frutos de leguminosas (FL) son de menor contenido de PC y EE; y altos contenidos de FDA, en comparación con los otros alimentos analizados. Mientras que se puede destacar que $\mathrm{AF}, \mathrm{ANL}, \mathrm{LNF}, \mathrm{PO}$ y SPR, presentan valores de NDT

Tabla I. Localización geográfica, área (ha) y clasificación de los sistemas de producción ovinos caracterizados, en el departamento de Córdoba (Geographical location, area (ha) and classification of characterized sheep production systems, in the department of Córdoba).

\begin{tabular}{|c|c|c|c|c|c|c|}
\hline Subregión & Municipio & Longitud $\mathrm{N}$ & Latitud $\mathrm{O}$ & Sistema de Producción & Altura msnm & Área en Ovinos (ha) \\
\hline \multirow{2}{*}{ Zona Costanera } & Los Córdoba & $08^{\circ} 50^{\prime} 00,0^{\prime \prime}$ & $076^{\circ} 20^{\prime} 37^{\prime \prime}$ & Semi intensivo & 90 & 6 \\
\hline & Pto. Escondido & $09^{\circ} 01^{\prime} 33,2^{\prime \prime}$ & $076^{\circ} 04^{\prime} 58,7^{\prime \prime}$ & Extensivo-b ${ }^{1}$ & 58 & 70 \\
\hline \multirow{4}{*}{ Sabana } & Chinú & $09^{\circ} 05^{\prime} 22^{\prime \prime}$ & $075^{\circ} 22^{\prime} 54,5^{\prime \prime}$ & Extensivo-b & 97 & 25 \\
\hline & Chinú & $09^{\circ} 3^{\prime} 25,9^{\prime \prime}$ & $075^{\circ} 28^{\prime} 22,6^{\prime \prime}$ & Extensivo-bb ${ }^{2}$ & 82 & 20 \\
\hline & Sahagún & $08^{\circ} 46^{\prime} 05,3^{\prime \prime}$ & $075^{\circ} 24^{\prime} 52,7^{\prime \prime}$ & Extensivo-b & 106 & 80 \\
\hline & Sahagún & $08^{\circ} 57^{\prime} 27,7^{\prime \prime}$ & $075^{\circ} 29^{\prime} 794^{\prime \prime}$ & Extensivo-b & 46 & 22 \\
\hline \multirow{2}{*}{ Alto Sinú } & Tierralta & $08^{\circ} 10^{\prime} 01,8^{\prime \prime}$ & $075^{\circ} 54^{\prime} 11,6^{\prime \prime}$ & Extensivo-bb & 97 & 60 \\
\hline & Tierralta & $08^{\circ} 14^{\prime} 46,2^{\prime \prime}$ & $075^{\circ} 53^{\prime} 36,4^{\prime \prime}$ & Extensivo-b & 59 & 200 \\
\hline \multirow{3}{*}{ Sinú Medio } & San Carlos & $08^{\circ} 42^{\prime} 07^{\prime \prime}$ & $075^{\circ} 43^{\prime} 20^{\prime \prime}$ & Extensivo & 26 & 4 \\
\hline & Ciénaga de Oro & $08^{\circ} 50^{\prime} 49^{\prime \prime}$ & $075^{\circ} 35^{\prime} 43,5^{\prime \prime}$ & Extensivo-b & 54 & 12 \\
\hline & Montería & $08^{\circ} 33^{\prime} 541^{\prime \prime}$ & $076^{\circ} 0^{\prime} 667^{\prime \prime}$ & Semi intensivo-b & 32 & 6 \\
\hline \multirow{9}{*}{ Bajo Sinú } & Momil & $09^{\circ} 13^{\prime} 57,5^{\prime \prime}$ & $075^{\circ} 38^{\prime} 34,8^{\prime \prime}$ & Extensivo-b & 20 & 80 \\
\hline & Momil & $09^{\circ} 18^{\prime} 42,1^{\prime \prime}$ & $075^{\circ} 38^{\prime} 07,1^{\prime \prime}$ & Extensivo-b & 45 & 5 \\
\hline & Momil & $09^{\circ} 14^{\prime} 19,3^{\prime \prime}$ & $075^{\circ} 36^{\prime} 32,3^{\prime \prime}$ & Extensivo-b & 48 & 200 \\
\hline & Momil & $09^{\circ} 13^{\prime} 41,3^{\prime \prime}$ & $075^{\circ} 39^{\prime} 16,1^{\prime \prime}$ & Extensivo-b & 21 & 40 \\
\hline & Momil & $09^{\circ} 14^{\prime} 03,8^{\prime \prime}$ & $075^{\circ} 39^{\prime} 03,8^{\prime \prime}$ & Semi Intensivo- $b$ & 21 & 3 \\
\hline & Momil & $09^{\circ} 14^{\prime} 35,7^{\prime \prime}$ & $075^{\circ} 41^{\prime} 28,2^{\prime \prime}$ & Extensivo-b & 21 & 32 \\
\hline & Momil & $09^{\circ} 14^{\prime} 32,5^{\prime \prime}$ & $075^{\circ} 41^{\prime} 12,6^{\prime \prime}$ & Extensivo-b & 21 & 15 \\
\hline & Momil & $09^{\circ} 16,8^{\prime} 93^{\prime \prime}$ & $075^{\circ} 39,1^{\prime} 34^{\prime \prime}$ & Semi intensivo & 38 & 2 \\
\hline & Chimá & $09^{\circ} 04^{\prime} 04,3^{\prime \prime}$ & $075^{\circ} 36^{\prime} 30,5^{\prime \prime}$ & Extensivo-b & 19 & 20 \\
\hline \multirow{6}{*}{ San Jorge } & Buenavista & $08^{\circ} 17^{\prime} 37,6^{\prime \prime}$ & $075^{\circ} 24^{\prime} 11^{\prime \prime}$ & Intensivo & 57 & 22 \\
\hline & Planeta Rica & $08^{\circ} 27^{\prime} 23,4^{\prime \prime}$ & $075^{\circ} 33^{\prime} 35^{\prime \prime}$ & Semi intensivo & 80 & 11 \\
\hline & Planeta Rica & $08^{\circ} 25^{\prime} 39,4^{\prime \prime}$ & $075^{\circ} 33^{\prime} 16,5^{\prime \prime}$ & Semi intensivo & 77 & 10 \\
\hline & Planeta Rica & $08^{\circ} 08^{\prime} 41,8^{\prime \prime}$ & $075^{\circ} 38^{\prime} 30^{\prime \prime}$ & Extensivo-b & 124 & 60 \\
\hline & Planeta Rica & $08^{\circ} 08^{\prime} 49,4^{\prime \prime}$ & $075^{\circ} 38^{\prime} 15^{\prime \prime}$ & Extensivo-b & 126 & 0,2 \\
\hline & Planeta Rica & $08^{\circ} 22^{\prime} 33,9^{\prime \prime}$ & $075^{\circ} 34^{\prime} 11,6^{\prime \prime}$ & Extensivo-b & 55 & 2 \\
\hline
\end{tabular}

${ }^{1}$ Extensivo: ovinos pastoreando sobre grandes áreas en tierras marginales, inapropiadas para otras formas de agricultura. ${ }^{2}$ Semi intensivo: combinación entre el sistema extensivo e intensivo, este representa la inversión en instalaciones y alimentos concentrados. Intensivo: alta población ovina en un área reducida con suplementación variada. ${ }^{3} \mathrm{~b}$ : unidad productiva con ovinos y bovinos, ${ }^{4} \mathrm{bb}$ : unidad productiva con ovinos, bovinos y búfalos. 
superiores al $62 \%$, lo cual es indicativo de su potencial nutricional. Adicionalmente, se resalta el aporte energético de la torta de palma de aceite perteneciente al grupo de las $\mathrm{PO}$ y SPR, RT, que son cultivos cuyas áreas están en aumento en la región Caribe.

En la Tabla III, se presenta la composición de minerales de los recursos alimenticios disponibles en las UP, evidenciando que el contenido de macrominerales (Calcio, Fósforo y Potasio) de las AFyANL es mayor que en los recursos alimenticios LF, OF, FL y GF. Con relación a la concentración de microminerales (Hierro, Manganeso y Zinc), la mayor concentración (mg/kg) la presentaron los recursos GF y OF.

\section{MANEJO DE LA ALIMENTACIÓN}

Las principales características de manejo de los sistemas de alimentación ovinos se presentan en la Figura 2. En los indicadores se observa el deficiente manejo de praderas que aunado a las restricciones de lluvias y riego para el desarrollo de las pasturas en la época seca, hace que se presente insuficiente oferta forrajera, se pierda condición corporal en los ovinos y aumente la edad al sacrificio de estos. No obstante, el 53,85\% de los productores utiliza algún tipo de suplementación

Tabla II. Composición de macro componentes y de energía disponible de los forrajes y otros recursos alimenticios usados en las unidades productivas caracterizadas en el departamento de Córdoba (Composition of macro components and available energy of fodder and other food resources used in the production units characterized in the department of Córdoba).

\begin{tabular}{|c|c|c|c|c|c|c|c|c|c|}
\hline Nutrientes (\%) & Estadística & ${ }^{1} \mathrm{GF}$ & ${ }^{2} \mathrm{OF}$ & ${ }^{3} \mathrm{FL}$ & ${ }^{4} \mathrm{AFyANL}$ & ${ }^{5} \mathrm{LNF}$ & ${ }^{6} \mathrm{PO}$ y SPR & ${ }^{7} \mathrm{RT}$ & ${ }^{8} \mathrm{Ml}$ \\
\hline \multirow[t]{2}{*}{ MS } & Promedio & 35,71 & 30,17 & 86,77 & 30,08 & 31,77 & 90,94 & 38,31 & 83,58 \\
\hline & Desv & 17,27 & 8,67 & 3,80 & 8,41 & 6,34 & 6,32 & & 23,34 \\
\hline \multirow[t]{2}{*}{ PC } & Promedio & 9,31 & 15,70 & 10,93 & 18,73 & 21,74 & 24,31 & 3,02 & 18,01 \\
\hline & Desv & 4,66 & 6,26 & 4,46 & 5,43 & 3,22 & 12,76 & & 5,49 \\
\hline \multirow[t]{2}{*}{ CNZ } & Promedio & 9,59 & 8,62 & 5,79 & 9,89 & 8,41 & 5,52 & 2,74 & 10,98 \\
\hline & Desv & 2,56 & 2,60 & 1,39 & 2,09 & 0,95 & 1,30 & & 5,85 \\
\hline \multirow[t]{2}{*}{ EE } & Promedio & 1,97 & 2,59 & 1,14 & 3,33 & 3,20 & 9,26 & & 8,08 \\
\hline & Desv & 0,61 & 1,53 & 0,57 & 1,50 & 0,55 & 3,14 & & 4,81 \\
\hline \multirow[t]{2}{*}{ FDN } & Promedio & 60,53 & 50,43 & 29,64 & 39,19 & 35,46 & 47,50 & 8,01 & 35,08 \\
\hline & Desv & 6,17 & 8,12 & 5,58 & 5,94 & 2,82 & 14,34 & & 7,79 \\
\hline \multirow[t]{2}{*}{ FDA } & Promedio & 34,06 & 26,65 & 12,12 & 19,30 & 14,93 & 29,38 & 3,55 & 25,34 \\
\hline & Desv & 6,07 & 5,27 & 6,48 & 5,94 & 4,87 & 8,19 & & 3,49 \\
\hline \multirow[t]{2}{*}{ LIG } & Promedio & 9,93 & 6,20 & 2,14 & 4,17 & 2,84 & 6,08 & 0,05 & ,98 \\
\hline & Desv & 2,74 & 1,82 & 1,64 & 1,82 & 1,14 & & & 0,52 \\
\hline \multirow[t]{2}{*}{ CHOS NE } & Promedio & 7,58 & 6,53 & 17,35 & 6,11 & 9,57 & 9,6 & 65,14 & 16,39 \\
\hline & Desv & 5,44 & 2,74 & 5,59 & 2,97 & 1,14 & & & 13,69 \\
\hline \multirow[t]{2}{*}{ CHOS Sol } & Promedio & 3,25 & 3,05 & 4,51 & 2,84 & 3,54 & 9,6 & 3,19 & 3,03 \\
\hline & Desv & 1,36 & 2,38 & 1,13 & 1,35 & 1,37 & 3,73 & & 1,63 \\
\hline \multirow[t]{2}{*}{ EB } & Promedio & 4,05 & 4,18 & 4,03 & 4,18 & 4,25 & 2,9 & 4,65 & 4,26 \\
\hline & Desv & 0,09 & 0,15 & 0,05 & 0,11 & 0,1 & 3,07 & & 0,13 \\
\hline \multirow[t]{2}{*}{ NDT } & Promedio & 52,46 & 58,08 & 4,03 & 62,39 & 65,84 & 63,27 & 90,88 & 67,43 \\
\hline & Desv & 6,58 & 5,41 & 0,05 & 4,99 & 1,84 & 8,44 & & 3,59 \\
\hline \multirow[t]{2}{*}{ EM } & Promedio & 1,88 & 2,09 & 3,05 & 2,29 & 2,41 & 2,34 & & 2,53 \\
\hline & Desv & 0,31 & 0,27 & 0,23 & 0,24 & 0,13 & 0,37 & & 0,16 \\
\hline \multirow[t]{2}{*}{ ENm } & Promedio & 1,03 & 1,23 & 2,08 & 1,42 & 1,53 & 1,46 & & 1,63 \\
\hline & Desv & 0,29 & 0,25 & 0,19 & 0,22 & 0,11 & 0,32 & & 0,14 \\
\hline \multirow[t]{2}{*}{ ENg } & Promedio & 0,47 & 0,66 & 1,41 & 0,83 & 0,93 & 0,87 & & 1,02 \\
\hline & Desv & 0,26 & 0,22 & 0,16 & 0,19 & 0,1 & 0,29 & & 0,12 \\
\hline
\end{tabular}

${ }^{1}$ Gramíneas forrajeras = GF ${ }^{2}$ Otros forrajes $=$ OF ${ }^{3}={ }^{4}$ Arboles forrajeros y Arbustos no Leguminosos $=$ AFyANL ${ }^{5}$ Leguminosas No Forrajeras $=$ LNF ${ }^{6}$ Plantas Oleaginosas Subproductos $=$ POSPR ${ }^{7}$ Raíces y Tubérculos $=$ RT ${ }^{8}$ Mezcla de Ingredientes $=$ MI. Materia seca (MS) Proteína cruda (PC) Cenizas (CNZ) Extracto etéreo (EE) Fibra en detergente neutro (FDN) Fibra en detergente acido (FDA) Lignina (LIG) Hemicelulosa (HEM) Carbohidratos no estructurales (CHOS NE) Carbohidratos solubles ( CHOS sol) Energía bruta (EB) Nutrientes digestibles totales (NDT) Energía metabolizable (EM) Energía neta de mantenimiento (ENm) Energía neta de ganancia (ENG). 
(ensilaje, heno, subproductos de la agroindustria, semillas de árboles) y el 92,1\% suplemento mineral.

Alimentación: Con base en los resultados, la alimentación de los ovinos se fundamenta en el pastoreo de gramíneas tropicales, siendo la más utilizada Colosuana (Botriochloa pertusa), la cual se usa en monocultivo $(46,15 \%)$ o asociada con otras especies, generalmente mezclados. El 69,2\% de los predios posee Colosuana (Botriochloa pertusa) 39\% Angleton (Dichanthium aristatum), 19,2\% Guinea (Megathyrsus maximus), 12\% Estre- lla (Cynodon nlemfuensis), y el 54,8 \% utiliza otros pastos en pequeñas proporciones, así: Masai (Megathyrsus maximus cv masai), Decumbens (Brachiaria decumben Braquipará (Brachiaria plantaginea), Suazia (Digitaria suazilandensis), entre otros.

\section{SISTEMAS DE PASTOREO Y MANEJO DE LA PRADERA:}

En las UP, los sistemas de pastoreo utilizados son el continuo, rotacional, semi-intensivo y el alterno, con una representación del $42 \%, 27 \%$, 8\% y 3\%, respectivamente. El número promedio de potreros es de

Tabla III. Contenido mineral de los alimentos consumidos por los ovinos en las unidades productivas caracterizadas en el departamento de Córdoba (Mineral content of food consumed by sheep in the production units characterized in the department of Córdoba).

\begin{tabular}{|c|c|c|c|c|c|c|}
\hline Mineral & Estadístico & GF & OF & $\mathrm{FL}$ & ÁFyANL & LF \\
\hline \multirow[t]{2}{*}{ Calcio (\%) } & Promedio & 0,44 & 0,77 & 0,48 & 1,15 & 0,97 \\
\hline & Desv & 0,15 & 0,2 & 0,09 & 0,23 & 0,11 \\
\hline \multirow[t]{2}{*}{ Fósforo (\%) } & Promedio & 0,22 & 0,31 & 0,12 & 0,33 & 0,28 \\
\hline & Desv & 0,09 & 0,19 & 0,05 & 0,1 & 0,06 \\
\hline \multirow[t]{2}{*}{ Magnesio (\%) } & Promedio & 0,21 & 0,27 & 0,19 & 0,32 & 0,29 \\
\hline & Desv & 0,04 & 0,09 & 0,03 & 0,06 & 0,03 \\
\hline \multirow[t]{2}{*}{ Potasio (\%) } & Promedio & 1,67 & 1,73 & 1,08 & 1,85 & 1,59 \\
\hline & Desv & 0,54 & 0,51 & 0,62 & 0,66 & 0,37 \\
\hline \multirow[t]{2}{*}{ Sodio (\%) } & Promedio & 0,01 & 0,01 & 0,01 & 0,01 & 0,01 \\
\hline & Desv & 0 & 0 & 0 & 0 & 0 \\
\hline \multirow[t]{2}{*}{ Azufre (\%) } & Promedio & 0,08 & 0,14 & 0,08 & 0,23 & 0,22 \\
\hline & Desv & 0,05 & 0,14 & 0,05 & 0,12 & 0,07 \\
\hline \multirow[t]{2}{*}{ Cobre $(\mathrm{mg} / \mathrm{kg})$} & Promedio & 5,18 & 6,52 & 3,5 & 7,03 & 7,31 \\
\hline & Desv & 1,69 & 1,97 & 1,13 & 1,96 & 1,24 \\
\hline \multirow[t]{2}{*}{ Hierro $(\mathrm{mg} / \mathrm{kg})$} & Promedio & 84,85 & 85,86 & 19,3 & 81,81 & 43,11 \\
\hline & Desv & 46,47 & 49,8 & 10,35 & 41,94 & 29,05 \\
\hline \multirow[t]{2}{*}{ Manganeso $(\mathrm{mg} / \mathrm{kg})$} & Promedio & 34,26 & 32,07 & 22,45 & 31,02 & 30,57 \\
\hline & Desv & 6,77 & 11,13 & 3,68 & 9,25 & 9,41 \\
\hline \multirow[t]{2}{*}{ Zinc (mg/kg) } & Promedio & 36,75 & 32,7 & 29,39 & 27,47 & 25,59 \\
\hline & Desv & 3,84 & 6,85 & 5,39 & 10,03 & 2,8 \\
\hline \multirow[t]{2}{*}{ Boro (mg/kg) } & Promedio & 13,57 & 26,25 & 19,97 & 36,34 & 38,68 \\
\hline & Desv & 3,96 & 13,52 & 3,95 & 9,55 & 3,25 \\
\hline
\end{tabular}

${ }^{1}$ Gramíneas forrajeras $=$ GF ${ }^{2}$ Otros forrajes $=$ OF ${ }^{3}$ Forrajes leguminosos $=F L{ }^{4}$ Arboles forrajeros y Arbustos no Leguminosos $=$ ÁFyANL ${ }^{5}$ Leguminosas Forrajeras $=$ LF.

8,8; con un área de 3,97 ha, manejados bajo un ciclo de pastoreo de 50,3 d (11,7 días de ocupación y 38,6 días de descanso). El 15,4\% de los productores estima la disponibilidad de biomasa de las praderas mediante aforos y ninguno determina la composición nutricional de los materiales forrajeros. Para el control de plagas y malezas los productores utilizan el $8 \%$ productos biológicos, $8 \%$ sistema integral (químico + biológico) y $4 \%$ no realiza control de plagas. El $28 \%$ de los productores ha realizado análisis de suelos, el 34,6\% fertiliza y lo realizan con productos químicos $(50 \%)$, productos biológicos u orgánicos $3,8 \%$ y con productos orgánicos + químicos el 3,8\%.

Suplementación: Los productores recurren al establecimiento de cultivos forrajeros para la suplementación de los animales en las épocas críticas. En este orden de ideas, el 42,3\% de los productores posee entre 0,12 a 4 ha en pastos de corte de especies Zea Mays, Pennisetum purpureum y los cosecha a los 80 días de rebrote.

La estrategia de suplementar a los animales en pastoreo la implementa el 53,85\% de los productores durante la época seca del año y dentro de estos, el 34,62\% suministra forrajes conservados (ensilaje, heno); el 3,85\% alimentos balanceados (subproductos de la agroindustria); $15,38 \%$ otras fuentes (semillas de árboles, grasa sobrepasante, fuentes nitrogenadas, bloques multinutricionales y concentrado).

La frecuencia de la práctica en la suplementación del rebaño, en las 26 UP varió según la categoría animal, presentándose suplementación en varios lotes de 
animales de algunas UP. El 50\% de las UP se suplementan las hembras paridas, mientras que el 14,29\% suplementa las hembras secas, el 28,57\% a las hembras de levante, el 35,71\% a las crías en lactancia, 28,57\% a machos de levante y $42,86 \%$ a los reproductores. Es de resaltar que el $42,86 \%$ de los productores que suplementan crías los hacen mediante el uso del creep feeding.

Suplementación mineral: el suministro de sal mineralizada a los ovinos es una práctica que usualmente es empleada en las UP (92,31\%) y solo el 8,69\% de ellas no suministra ningún tipo de suplemento mineral. Con relación al tipo de suplemento mineral, el 45,83\% de los productores suplementa con sal mineralizada formulada para bovinos, mientras que el 54,17\% suministra sal específicamente balanceada para ovinos, el consumo promedio oscila entre 5 y 32 gramos por animal al día.

Suministro de agua: la fuente de agua utilizada para el consumo de los animales en las UP es propor-

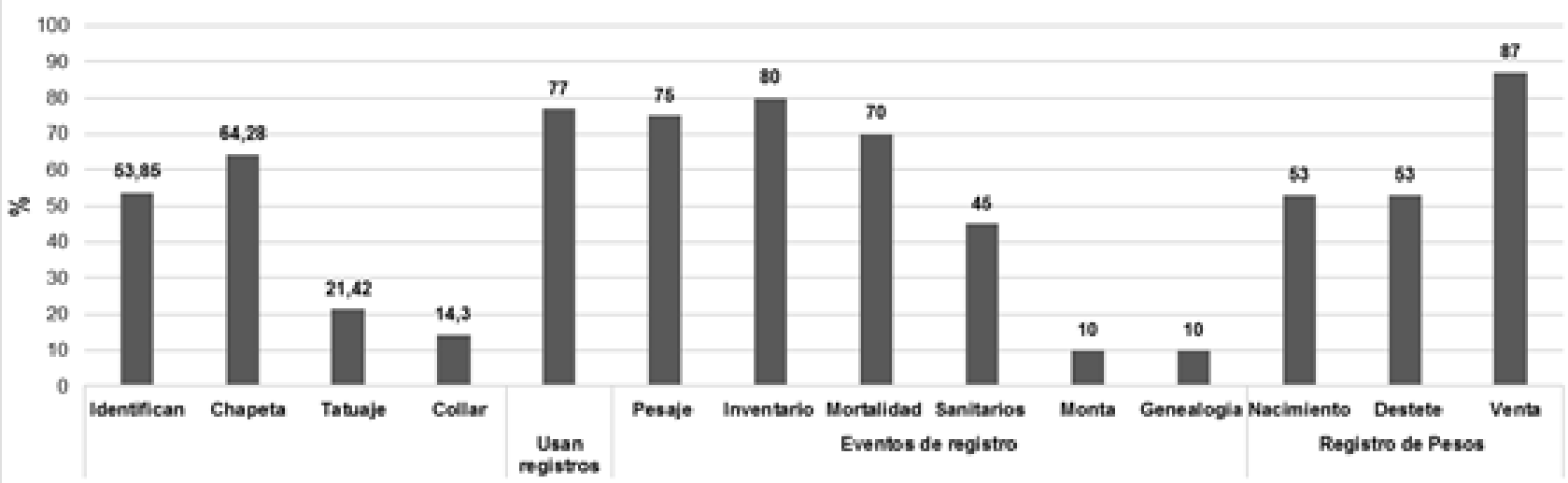

Figura 1. Características del Manejo y control de ovinos en unidades productivas del departamento de Córdoba (Characteristics of sheep management and control in production units of the department of Córdoba).

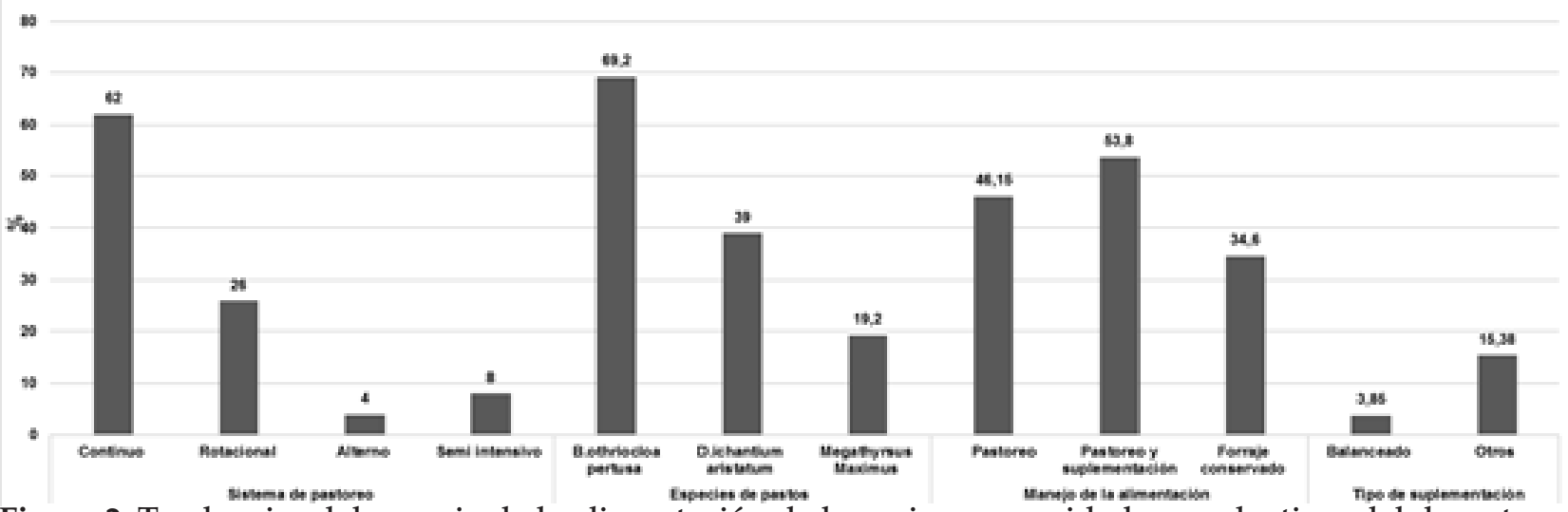

Figura 2. Tendencias del manejo de la alimentación de los ovinos en unidades productivas del departamento de Córdoba (Trends in the management of sheep feed in production units in the department of Córdoba).

cionada principalmente $(61,53 \%)$ por estaques artificiales o jagüey, mientras que el 19,23\% $15,38 \%$ y $3,85 \%$ es proveniente de pozos profundos, caños o quebradas y acueducto, respectivamente.

\section{RESULTADOS DEL AGRUPAMIENTO DE FINCAS}

Desde el ACM, se obtuvo que para explicar el 73.4\% de la inercia total, fue necesario retener 8 ejes (variables sintéticas), los cuales fueron combinados con las variables cuantitativas para llevar a cabo el análisis de conglomerados. El número óptimo de grupos, para el método de las $\mathrm{K}$ medias fue de dos, mientras que, para el agrupamiento jerárquico fue tres. La correlación cofenética obtenida fue de 0.67 , lo que indica que las distancias originales no fueron conservadas en el dendograma. Considerando el criterio de Dunn y la amplitud de silueta, el agrupamiento jerárquico tuvo un índice de Dunn de 0,23 y una media para la amplitud de silueta de 0,24 , mientras que el método de las $\mathrm{K}$ medias tuvo un índice de Dunn de 0,32 y amplitud de silueta media igual a 0,51 . En cuanto a las UP con amplitud de silueta negativa como indicador de errores en la clasificación por parte del método (o UP que se encuentran entre dos grupos), para el método jerárquico, resultaron siete UP con amplitud negativa y para las K medias solo una UP con amplitud negativa.

De acuerdo con los resultados de los criterios de validación interna utilizados, el método K medias presenta la mejor estructura de agrupamiento en comparación con el método jerárquico, por tanto, los resultados de caracterización presentados se centran en la descripción de las 26 UP agrupadas en 2 conglomerados, denominados como G1 y G2, respectivamente. 
Tabla IV. Frecuencias (\%) de las variables cualitativas evaluadas en las UP del departamento de Córdoba,

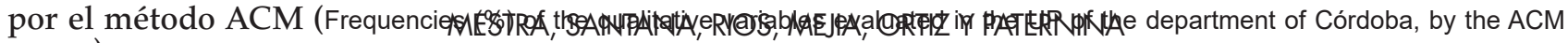
method).

\begin{tabular}{|c|c|c|c|}
\hline \multirow[t]{2}{*}{ Variable } & \multirow[t]{2}{*}{ Categoría } & $\underset{1}{\text { Grupo }}$ & $\begin{array}{c}\text { Grupo } \\
2\end{array}$ \\
\hline & & $(\%)$ & $(\%)$ \\
\hline \multirow{7}{*}{ Subregión } & Alto Sinú & 33,3 & 4,4 \\
\hline & Medio Sinú & 0,0 & 8,7 \\
\hline & Bajo Sinú & 0,0 & 39,1 \\
\hline & Costanera & 33,3 & 4,4 \\
\hline & San Jorge & 33,3 & 21,7 \\
\hline & Sabanas & 0,0 & 17,4 \\
\hline & Centro & 0,0 & 4,4 \\
\hline \multirow{6}{*}{ Tipo de identificación } & Chapeta & 66,7 & 30,4 \\
\hline & Microchip & 0,0 & 0,0 \\
\hline & Collar & 0,0 & 8,7 \\
\hline & Tatuaje & 0,0 & 13,0 \\
\hline & Tatuaje + Chapeta & 0,0 & 21,7 \\
\hline & Ninguno & 33,3 & 26,1 \\
\hline \multirow{9}{*}{ Registros } & Sanitario & 33,3 & 34,8 \\
\hline & Pesaje & 66,7 & 56,5 \\
\hline & Montas & 66,7 & 0,0 \\
\hline & Muertes & 100,0 & 52,2 \\
\hline & Inventario & 66,7 & 73,9 \\
\hline & Genealogía & 0,0 & 13,0 \\
\hline & Peso al nacimiento & 66,7 & 34,8 \\
\hline & Peso al destete & 100,0 & 34,8 \\
\hline & Peso a la venta & 66,7 & 78,3 \\
\hline \multirow{5}{*}{ Tipo de pasto } & Colosuana & 33,3 & 73,9 \\
\hline & Estrella & 33,3 & 8,7 \\
\hline & Angleton & 33,3 & 39,1 \\
\hline & Guinea & 33,3 & 17,4 \\
\hline & Otros & 66,7 & 52,2 \\
\hline \multirow{4}{*}{ Tipo de pastoreo } & Continuo & 66,7 & 60,9 \\
\hline & Rotacional & 0,0 & 30,4 \\
\hline & Alterno & 0,0 & 4,4 \\
\hline & Semi-intensivo & 33,3 & 4,4 \\
\hline \multirow{5}{*}{ Tipo de suplemento } & No suplementa & 33,3 & 47,8 \\
\hline & Forrajes conservados & 33,3 & 21,7 \\
\hline & Subprod Agro-industria & 0,0 & 13,0 \\
\hline & Forrajes conservados + Subprod Agro-industria & 0,0 & 4,4 \\
\hline & Forrajes conservados + Grasa sobrepasante+BMN+Concentrados & 33,3 & 13,0 \\
\hline
\end{tabular}

En la Tabla IV se pueden ver las frecuencias (\%) obtenidas para cada una de las categorías de respuesta de las preguntas de tipo cualitativo.

Ubicación: Como se observa en la Tabla IV, las UP agrupadas en G1 se distribuyen equitativamente en las subregiones Alto Sinú, Zona Costanera y San Jorge, mientras que las UP del G2 se distribuyen de manera más heterogénea en todas (7/7) las subregiones del departamento, destacándose que aproximadamente el $40 \%$ de estos predios pertenecen al Bajo Sinú.
Identificación: En general el método más utilizado en las UP para la identificación de los ovinos es la chapeta. En las UP del G1, el 66,7\% de los productores identifica los ovinos y lo hace mediante este método, mientras que el porcentaje restante no identifica sus animales (33,3\%). Por otra parte, en el G2 el 77,9\% identifica con una mayor variedad de formas.

Uso y manejo de registros: En general, se destaca que el G1 utiliza con mayor frecuencia los registros en comparación con las UP del G2. De esta manera, en el 
G1 la totalidad de las UP lleva registros de mortalidad y peso al destete, mientras que, en el G2, la frecuencia de uso de estos registros es inferior, para este grupo la prioridad es dada a los registros de inventario y peso a la venta.

Tipos de pastos: en el G1 el uso de los pastos (Colosuana, Estrella, Angleton y Guinea) es proporcional en las distintas UP, adicionalmente, la mayoría de estos predios $(66,7 \%)$ poseen otros pastos en áreas pequeñas. En el G2 predomina el uso del pasto Colosuana, y aproximadamente mitad de estas UP utilizan áreas pequeñas con otros pastos para complementar la alimentación de los animales.

Tipos de pastoreo: En cuanto a los tipos de pastoreo, predomina en ambos grupos el pastoreo continuo, en segundo lugar, el de mayor frecuencia para el G1 es el pastoreo semi-intensivo, mientras que para el G2 es el rotacional, encontrándose además en este grupo con menor frecuencia, otros sistemas de pastoreo.

Tipos de suplementos: Se encontró que en el G1 los tipos de suplementos ofrecidos son menos diversos que los del G2, sin embargo, la frecuencia de suplementación de este grupo es menor.

Estructura del rebaño: La población total en promedio para las UP en cada grupo era de 422 animales en los rebaños del G1 y 90,6 animales en las UP del G2. Mientras que la distribución de los distintos estados fisiológicos dentro de los rebaños evaluados fue la siguiente, para el G1 las hembras adultas representan $45,2 \%$, hembras de reemplazo $13,3 \%$, corderos $39,9 \%$ y machos reproductores $1,6 \%$. De otro lado, para las UP del G2 las categorías se distribuían de la siguiente manera: hembras adultas representan $43,2 \%$, hembras de reemplazo $15,1 \%$, corderos $39,4 \%$ y machos reproductores $2,3 \%$. Con relación al número de crías por oveja parida se encontró que en ambos grupos de UP (G1 y G2), el número era de 1,1 cordero por oveja.

\section{DISCUSIÓN}

Las razas de ovinos más frecuentemente encontradas en las UP eran del tipo OPC (Ovino de pelo colombiano), las cuales son adaptadas a las condiciones de alta humedad y temperatura características de esta zona, con importante resistencia a enfermedades, tamaño mediano y carne con bajo contenido en grasa. De otro lado, en Colombia, desde el año 2002 se han importado razas especializadas para la producción de carne tales como: Dorset, Suffolk, Romanov, Kathadin, Dorper y Pelibuey, las cuales son cruzadas con los animales locales sin ningún tipo orientación (Martínez-Correal, 2010). De manera contraria, en el estudio de Moreno y Grajales (2017), encontraron en las UP estudiadas por ellos en el trópico alto colombiano, que las razas más comunes resultaron ser Romney Marsh, Hampshire, Corriedale y Criolla y en los rebaños se realizaba el pesaje como una práctica común usando la báscula de reloj: en los sistemas carne y lana en un $77 \%$, en los de genética y carne en un $44 \%$ y en los de solo carne en un $64 \%$.

En cuanto a la asociación de las especies ovina y bovina, tiene mayor presencia en las UP caracteriza- das en el Caribe húmedo (96\%) que en el Caribe seco colombiano, en un estudio reciente (Rúa-Bustamante et al., 2018), destacan que en el departamento del Cesar el $52 \%$ de los productores asocia actividades. Así mismo, en estudios desarrollados (Cadena Productiva OvinoCaprina Nacional, 2012), en el acuerdo de competitividad para la cadena productiva ovina en Colombia, se reportó que el 50\% de los productores cuenta con otros sistemas de producción pecuarios. Este tipo de sistemas de producción mixtos son muy usados en países con mayor tradición en producción ovina que Colombia, como es el caso de Uruguay y México, debido a la gran cantidad de ventajas que presenta, entre las cuales se pueden enumerar: mejora el uso de los forrajes, mejor control de malezas y parásitos, aparte de aumentar el ingreso por unidad de superficie (Sharpe, 2019), De esta manera, en el estudio de Mendiola-González et al. (2007), se señalan que se presenta mayor el aprovechamiento del forraje ( $24 \%$ menos forraje residual) cuando pastoreaban las dos especies de manera conjunta (terneras y ovejas).

Con relación a la estructura de los rebaños estudiados (G1 y G2) se encontró una distribución similar entre los dos grupos del presente estudio, y a su vez semejante a la señalada por Orona-Castillo et al. (2014), como representativa y económicamente viable, en un estudio realizado en México, que constaba de aproximadamente de 50\% hembras adultas, 10\% hembras de reemplazo, $40 \%$ de corderos y $1 \%$ de machos reproductores.

Con respecto a la toma de registros en las UP, es importante fortalecer el uso de estos, ya que son la base para evaluaciones de indicadores de crecimiento y mejoramiento genético (Agudelo-Gómez, CerónMuñoz y Restrepo, 2007). Luego del paso de fortalecer la implementación de registros en las UP, se puede pensar en usar software para el control del rebaño, ya que permite la toma de decisiones correctas dentro de las mismas, actualmente en Colombia el uso de softwares es bajo (González et al., 2011).

En las UP estudiadas la asistencia técnica, la identificación y el manejo de registros individuales en las fincas, es de uso más común, que el encontrado en el departamento del Cesar, donde solo el 30\% accede a la asistencia técnica, solo el 3\% lleva registros y el 66\% no identifica los semovientes ovinos (Rúa-Bustamante et al., 2018).

El peso al destete encontrado en el presente estudio es similar al reportado por Rúa-Bustamante (2015) para la raza Kathadin $(17 \mathrm{~kg})$ y mayor al señalado para OPC (9 kg); estando en el rango señalado por Martínez y Malagón (2005) y por debajo de lo esperado en el acuerdo de competitividad en el cual se propone destete a 60 días con 15 kg (Cadena Productiva OvinoCaprina Nacional, 2012). En los sistemas de producción ovina de trópico alto (Moreno y Grajales, 2017) encontraron pesos al destete de 6 a $20 \mathrm{~kg}$ en sistemas de carne y en los de carne y lana, mientras que el rango era de 20 a $34 \mathrm{~kg}$ en los sistemas dedicados a genética y carne, en todos los casos la edad al destete estaba en el rango de 2 a 5 meses. Un promedio de $16 \%$ de los productores 
de los distintos sistemas no conocen la edad al destete de sus crías.

En cuanto a la proporción promedio de corderos por oveja parida se señaló que para ambos grupos era de 1,1 lo cual pudiera ser una ventaja en estos sistemas extensivos, debido al limitado manejo en general que reciben los corderos en este tipo de sistemas, teniendo en consideración que las crías, productos de partos simples presentan crías de mayor peso, y en consecuencia mayor probabilidad de supervivencia hasta el destete. Coincidiendo con los resultados de Lima et al., (2019) quienes señalaron mayor sobrevivencia de los corderos de partos simple en comparación con los de partos dobles hasta los 100 días de edad.

Respecto a la alimentación y la suplementación del rebaño, se encontró que un alto porcentaje de los productores usan recursos alimenticios distintos al pasto para complementar la alimentación de sus animales, coincidiendo esto con lo expresado por Castellanos et al., (2010) quienes señalaron que el rubro de alimentación representa aproximadamente $59 \%$ de los costos de producción en las UP de mediana a alta tecnología, ya que los de bajo nivel no reportan compra de alimentos. Por otra parte, siendo el pastoreo de tipo extensivo en el $62 \%$ de las UP, resulta relevante mencionar un estudio de Vilaboa-Arroniz et al., (2006), donde encontraron que los sistemas de producción semi-intensivos producían más carne por unidad de superficie cuando se comparaba con el sistema de producción extensivo, pero con una menor autonomía. El manejo de la suplementación de los ovinos de levante es similar a la de bovinos ya que un reducido número de productores los suplementan, y para una alimentación eficiente en la zona de estudio, es indispensable que todos los ovinos reciban forrajes de corte, frescos o conservados y/o suplementos especialmente en la época de sequía debido que se presentan déficit de materia seca de buena calidad para los ovinos (Maza-Angulo et al., 2015 y Vergara-Garay et al., 2017). Estudios de Cajas-Girón et al., (2005) indican que en cada unidad productiva coexisten varias especies forrajeras, lo que permite a los ovinos obtener alimento durante todo el año, siendo principalmente frutos y rebrotes de arbustivas en época de sequía ya que el pasto predominante es Colosuana que presenta reducciones en su disponibilidad hasta del $83 \%$ en esta época.

\section{CONCLUSIÓN}

En el departamento de Córdoba las unidades productivas de ovinos en su mayoría son extensivas, manejadas en pastoreo continuo con un bajo nivel de adopción de tecnologías y mediano control de sus animales mediante registros.

En las unidades productivas caracterizadas en el departamento de Córdoba, la producción ovina es una actividad practicada en pastoreo únicamente o con suplementación, en un proceso de transición de un nivel tecnológico bajo a medio, existiendo unidades productivas muy organizadas y otras donde los ovinos no reciben atención como factores de producción.
La producción ovina en Córdoba en su mayoría se desarrolla en asociación con la producción bovina que es la principal actividad económica del departamento, condición que es similar a otras regiones del país.

De acuerdo con el análisis por componentes principales se diferenciaron 2 grupos de UP, el G1 con rebaños grandes (entre 260 y 600 animales) y el G2 de rebaños más pequeños ( $<100$ animales), encontrándose en ambos que predomina el pastoreo continuo y uso de suplementos de manera estratégica la cual se presenta con menor frecuencia en el G2, destacándose también una buena estructura de los rebaños, por estados fisiológicos de los animales en ambos grupos, y así también una relación de número de crías por oveja parida acorde al manejo del sistema.

Existe una minoría de productores dedicados exclusivamente a la ovinocultura en sistema semi extensivo con uso de asistencia técnica, mayor inversión en infraestructura y pie de cría lo cual constituye una oportunidad para adelantar estudios que permitan integrar la ovinocultura y la agroindustria para aumentar su productividad, contribuyendo así al uso racional de recursos y a la producción de carne de buena calidad para consumo nacional e internacional.

\section{AGRADECIMIENTOS}

Los autores agradecen a la Corporación Colombiana de Investigación Agropecuaria (AGROSAVIA), y Ministerio de Agricultura y Desarrollo Rural (MADR) por el soporte logístico y financiero dado durante la ejecución de esta investigación, desarrollada en el marco de la agenda corporativa nacional.

\section{BIBLIOGRAFÍA}

Acero-Plazas, V. M. (2014) 'El bienestar animal en sistemas productivos de ovinos-caprinos en Colombia', Spei Domus, 10(21), pp. 57-62. doi: https://doi.org/10.16925/sp.v10i21.918.

Agrosavia (2020) AlimenTro, Resultados Análisis Caesalpinia coriaria. Available at: https://alimentro.agrosavia.co/Estadisticas/ ReporteAnalisis.

Agudelo-Gómez, D. A., Cerón-Muñoz, M. and Restrepo, L. F. (2007) 'Modelación de funciones de crecimiento aplicadas a la producción animal', Revista Colombiana de Ciencias Pecuarias, 20, pp. 157-173. Available at: https://revistas.udea.edu.co/index.php/rccp/article/ view/324133.

Aguilar-Barojas, S. (2005) 'Fórmulas para el cálculo de la muestra en investigaciones de salud', Salud en Tabasco, 11(1-2), pp. 333-338.

AOAC International (1990) Official Methods of Analysis. Arlington, Virginia, USA.

Arcos, J. C. etal. (2002) Ovinos Colombianos de Pelo. Alternativa productiva para el sur del Departamento del Tolima, hitps://repository.agrosavia.co/bitstream/handle/20.500.12324/12831/42379_46150. pdf? sequence $=1$ \& isAllowed $=y$.

Arévalo-Garay, Á. and Correa-Assmus, G. (2013) 'Tecnología en la ovinocultura colombiana: Estado del arte', Revista Ciencia Animal, 6, pp. 125-142. Available at: https://www.semanticscholar.org/ paper/Tecnología-en-la-ovinocultura-colombiana\%3A-estado-GarayAssmus/aa5d66661 af95079b328d99el eb81 c2f9eabcc96.

Ariza-Nieto, C. et al. (2018) 'Use of LOCAL algorithm with near infrared spectroscopy in forage resources for grazing systems in Colombia', Journal of Near Infrared Spectroscopy, 26(1), pp. 44-56. doi: $10.1177 / 0967033517746900$. 
Cadena Productiva Ovino-Caprina Nacional (2012) 'Acuerdo Nacional DeCompetitividad', Acuerdo Nacional De Competitividad, pp. 1-89.

Cajas-Girón, Y. etal. (2005) Desarrollo e implementación de estrategias tecnológicas para mejorar la productividad y sostenibilidad de sistemas de doble propósito en las sabanas de la Región Caribe-Reporte final de proyecto. Montería. doi: 10.13140/RG.2.1.3836.6885.

Castellanos, J. G. et al. (2010) Agenda prospectiva de investigación y desarrollo tecnológico para la cadena productiva cárnica ovinocaprina en Colombia. Bogota.

FAO and IDF (2014) WORLD MAPPING OF ANIMAL FEEDING SYSTEMS IN THE DAIRY SECTOR. Rome, Italy. Available at: http://www. fao.org/3/a-i3913e.pdf.

González-Garduño, R., Torres-Hernández, G. and Arece-García, J. (2010) 'Comportamiento productivo y reproductivo de ovinos Pelibuey en un sistema de pariciones aceleradas con tres épocas de empadre al año', Zootecnia Tropical, 28(1), pp. 51-56. Available at: http://ve.scielo.org/scielo.php? script=sci_arttext\&pid $=$ S0798-72692010000100007.

González, C. A. et al. (2011) 'Gestion de la información en los sistemas de producción animal-Una mirada al caso de la ovino-caprinocultura', Rev. Med. Vet. Zoote., 58(III), p. 176:193.

Grajales, H. A., Moreno, D. C. and Atuesta-Bustos, J. E. (2011) Guía Técnica de Producción Ovina y Caprina: I. Aspectos favorables y desfavorables para la producción ovina y caprina. Bogotá, Colombia. Available at: https://repository.agrosavia.co/ handle/20.500.12324/34285.

Herrman, T. (2001) Sampling: Procedures for Feed. Kansas; USA. Available at: https://bookstore.ksre.ksu.edu/pubs/mf2036.pdf.

Holdridge, L. R. (1967) Life Zone Ecology. San José, Costa Rica.

ICA (2020) Censo Nacional Equinos-Caprinos-Ovinos-CENSOS-2020. Available at: https://www.ica.gov.co/areas/pecuaria/servicios/ epidemiologia-veterinaria/censos-2016/censo-2018.

IGAC (2009) Estudio general de suelos y zonificación de tierras del departamento de Córdoba, Instituto Geográfico Agustín Codazzi (IGAC). Edited by I. D. Gómez-Guzmán et al. Bogotá: Imprenta Nacional de Colombia.

Lima, M. Z. etal. (2019) 'Genetic and non-genetic analysis of lamb survival in Sangsari sheep by gibbs sampling method', Small Ruminant Research, 177(January), pp. 56-60. doi: 10.1016/i.smallrumres.2019.06.013.

Mahecha, L. etal. (2000) Consumo de pasto estrella africana (Cynodon plectostachyus) y leucaena (Leucaena leucocephala) en un sistema silvopastoril, Pasturas Tropicales. Available at: http://tropicalgrasslands.info/index.php/tgft/pages/view/Pasturas.

Martínez-Correal, G. (2010) Situación del recursos ovino y caprino en Colombia: documento de trabajo elaborado en el marco del plan nacional de acción para la conservación mejoramiento y utilización sostenible de los recursos genéticos animales de Colombia. Bogotá-Colombia: FAO

Martínez, R. and Malagón, S. (2005) 'Caracterización fenotípica y genética del ovino criollo colombiano', Archivos de Zootecnia, 54(206-207), pp. 341-348.

Maza-Angulo, L. et al. (2015) 'Efecto de la suplementación sobre la ganancia de peso y rendimiento en canal de corderos Sudan', Revista UDCA Actualidad y Divulgación Científica. Bogotá, Colombia, 18(1), pp. 283-286. doi: 10.31910/rudca.v18.n1.2015.448.

Mendiola-González, A. et al. (2007) 'Efecto del pastoreo mixto y monoespecífico en una pradera de alfalfa-ovillo', Agrociencias, 41, pp. 395-403.

Montes, D., Pérez, A. and de la Ossa, J. (2016) 'Importancia de los ovinos en los sistemas de producción pecuaria_Perspectiva', in Revista Colombiana de Ciencia Animal, pp. 5-6.

Moreno, D. C. and Grajales, H. A. (2017) 'Caracterización de los sistemas de producción ovinos del trópico alto en Colombia: Manejo e Indicadores', Rev. Med Vet Zoot., 64(3), pp. 36-51. doi: 10.15446/ rfmvz.v64n3.68693.

Orona-Castillo, I. et al. (2014) 'Análisis microeconómico de una unidad representativa de producción de carne de ovino en el estado de México bajo un sistema de producción semi-intensivo', Revista Mexicana de Agronegocios, 34(enero-junio), pp. 720-728.

Owen, E. et al. (2004) Responding to the livestock revolution: The role of globalisation and implications for poverty alleviation. Nottingham, UK: Nottingham University Press. Available at: https://hdl.handle. net/10568/2315.

Palencia-Severiche, G., Mercado-Fernández, T. and Combatt-Caballero, E. (2006) Estudio agroclimático del departamento de Córdoba. Montería, Córdoba, Colombia: Universidad de Córdoba.

Pastrana-Bonilla, R. (2002) 'Ovinocultura colombiana su origen y evolución', in García-Alzate, H. and Parra-López, L. G. (eds) Medicina Veterinaria y Zootecnia en Colombia, Trayectoria durante el siglo XX y Perspectivas para el siglo XXI. lera edn. Bogotá-Colombia: Fundación Edivez, pp. 445-460. Available at: http://medicinaveterinariaydezootecnia.bogota.unal.edu.co/publicaciones/detalle. $\mathrm{html}$ ?tx_tnews\%5Btt_news\%5D=74\&cHash=6890bce02de24651 d7 fdofafb64a323f.

Pugh, D. G. and Baird, A. N. (2012) Sheep and Goat Medicine, 2nd Edition. 2nd edn. Edited by D. G. Pugh and A. N. Baird. Amsterdam, Netherlands: Elsevier. Available at: https://www.elsevier.com/books/ sheep-and-goat-medicine/baird/978-1-4377-2353-3.

Rodríguez-Ramírez, M. R. et al. (2013) 'Composición química de recursos forrajeros para la alimentación de ovinos en Colima'. Tecomán, Colima, México, p. 59 p. Available at: file:///D:/Descargas/silo. tips_composicion-quimica-de-recursos-forrajeros-para-la-alimentacion-de-ovinos-en-colima (1).pdf.

Rúa-Bustamante, C. V. (2015) Manual Técnico para la Producción de Carne Ovina Utilizando Buenas Prácticas Ganaderas. Medellin, Antioquia-Colombia. Available at: https://isbn.cloud/978958871 1751/ manual-tecnico-para-la-produccion-de-carne-ovina-utilizando-buenapracticas-ganaderas/.

Rúa-Bustamante, C. V. et al. (2018) 'Cadena de valor ovino caprina de la región Caribe seco de Colombia: 1. Diagnóstico de los sistemas de producción ovino caprina del departamento del Cesar', in (ALPA) (ed.) XXVI Reunión de la Asociación Latinoamericana de Producción Animal V Simposio Internacional de Producción Animal Guayaquil (Ecuador) 28 - 31 mayo, 2018. Guayaquil, Ecuador: Asociación Latinoamericana de Producción Animal, pp. 132-133.

Sharpe, P. (2019) 'Mixed species grazing', in Horse Pasture Management. Academic Press, pp. 157-175. doi: https://doi.org/10.1016/ B978-0-12-812919-7.00010-X.

Vergara-Garay, O. et al. (2016) 'Descripción del crecimiento en ovinos criollos utilizando el modelo Brody Using the Brody model for describing creole sheep growth Descrição do crescimento em ovinos crioula mediante o modelo de Brody', Orinoquia, 20(2), pp. 34-39.

Vergara-Garay, O. et al. (2017) 'Determinación de la curva de crecimiento en ovinos criollos de pelo, mediante la utilización del modelo Gompertz, en el trópico bajo colombiano', Revista UDCA Actualidad \& Divulgación Cientifica, 20(2), pp. 385-391.

Vilaboa-Arroniz, J. et al. (2006) 'Productividad y autonomía en Sistemas de Producción Ovina: Dos propiedades emergentes de los agroecosistemas', Interciencia, 31(1), pp. 38-44. 